\title{
REVISTA PERSPECTIVAS:
}

Estudios Sociales y Educación Cívica

ISSN-L: 2215-4728

DOI: http://dx.doi.org/10.15359/rp.20.2

http://www.revistas.una.ac.cr/perspectivas

N. ${ }^{\circ} 20$. Enero-Junio, $2020 \bullet$ pp. 1-27

\section{La enseñanza de los Estudios Sociales en la era de la calidad educativa}

\author{
Social Studies' Teaching in the Era of Quality \\ Education \\ David González Sánchez*
}

\section{Fecha de recepción: 04/10/2019 • Fecha de aceptación: 10/02/2020}

Resumen: Este ensayo explora los principios básicos del discurso de la calidad educativa y el modelo basado en habilidades que centra la importancia en el aprendizaje en tanto unidad de medida del éxito educativo. Argumento que esa visión tienen impacto en la enseñanza de los estudios sociales y, por consiguiente, en la forma como, desde la asignatura, se plantea la relación entre saber histórico, ciudadanía y pensamiento. Para respaldar estas ideas reviso la política educativa vigente, el currículo nacional y el programa de estudios de la asignatura. El resultado es una caracterización breve sobre tres cuestiones: la desvirtuación del pensamiento relegado a las habilidades, la confusa fundamentación teórica del programa para adaptar categorías como tiempo histórico y espacio geográfico a las exigencias del
Abstract: This essay explores the basic principles of quality education's discourse and the skill-based learning model and the impact on social studies subject-matter and therefore on the way in which the subject considers the relationship between historical knowledge, citizenship and thought. To support these ideas, I review the current education policy, the national curriculum and the curriculum of the subject. The result is a brief characterization on three issues: the distortion of thought relegated to skills, the confusing theoretical foundation of the program to adapt categories such as historical time and geographical space to the demands of the skills curriculum and the technification of learning.

* Costarricense. Máster en Estudios Latinoamericanos por la Universidad Nacional (UNA), Costa Rica y Licenciado en la Enseñanza de los Estudios Sociales y la Educación Cívica por la UNA, Costa Rica. Académico de la Escuela de Historia de la Universidad Nacional (UNA), Costa Rica. Correo electrónico: degs05@gmail.com. ORCID: https://orcid.org/0000-0002-4820-2803. 
Revista Perspectivas:

Estudios Sociales y Educación Cívica currículo en habilidades y la tecnificación del aprendizaje.

Palabras clave: Estudios Sociales; pedagogía; calidad; historia; aprendizaje.
Keywords: Social Studies; pedagogy; learning; history; quality.

\section{Introducción}

$\mathrm{E}$

n la agenda mundial sobre educación, al menos desde la década de los noventa del siglo XX, uno de los temas más álgidos ha sido el concerniente a la calidad educativa. Este concepto se caracteriza por multiplicidad de definiciones, en este escrito se refiere al conjunto de políticas educativas que denotan adscripción a las teorías del capital humano y a las exigencias de empleabilidad del mundo empresarial y que, en su dimensión más amplia, implica un cambio cultural que redefine el tipo de sociedad, el pensamiento y la función de la educación. La legitimidad del concepto se debe a la voluminosa producción de conocimientos de diversos organismos nacionales e internacionales y la incorporación de conceptos propios de tradiciones pedagógicas progresistas como la escuela nueva y la pedagogía crítica, que parece le han otorgado neutralidad a la expresión de calidad.

Mientras, operativamente, la calidad educativa establece los criterios de eficiencia, pertinencia y relevancia de los saberes por aprender, en función de la noción de productividad determinada por evaluaciones estandarizadas, lo que posibilita calcular tanto los niveles de aprendizaje y la retribución económica de la inversión en educación. Consecuentemente en Costa Rica, entre 2005 y 2018, el sistema educativo ha implementado una serie de reformas de la política educativa y el currículo nacional en primaria y secundaria. Muchas de las reformas han estado, en menor o mayor medida, guiadas por el discurso de la calidad educativa promovido desde el Banco Mundial (BM), la Organización de las Naciones Unidas para la Educación, la Ciencia y la Cultura (UNESCO) y la Organización para la Cooperación y el Desarrollo Económicos (OCDE). En consonancia, se han generado planes de estudio 
en los cuales el aprendizaje se ha ajustado al desarrollo de habilidades mediante indicadores que puedan ser evaluados a escala global. ${ }^{1}$

En ese contexto, la enseñanza de los estudios sociales ha empezado un proceso de mutación cargado de antinomias entre la ilusión de una supuesta renovación de la asignatura, el ajuste al modelo de habilidades y una dislocación del pensamiento social por la instrumentalización que implica el concepto de habilidades. A esto se le deben agregar los problemas que la asignatura ha venido arrastrando desde hace décadas en cuanto a la naturaleza del tipo de conocimiento que se pretende construir desde la enseñanza, los contenidos y una didáctica específica.

Este artículo pregunta por la relación e incidencia que han tenido las reformas recientes (2016-2018) a la política educativa y al currículo escolar costarricense con el signo de la calidad educativa sobre la enseñanza y el aprendizaje de los estudios sociales: ¿qué se transforma y qué queda en el plan de estudios de la asignatura?, ¿qué tipo de saber social plantean?, ¿qué ciudadanía pretende?, ¿qué papel puede jugar la didáctica de las ciencias sociales en este contexto de transformación epistemológica del currículo escolar?

El argumento propuesto para ensayar algunas respuestas es que los retos de la enseñanza de los estudios sociales deben leerse en el contexto de la hegemonía del discurso de la calidad educativa global y de las antinomias propias del lugar que se le otorga al pensamiento social en la escuela actual, inscrita en el modelo del desarrollo de habilidades. En este sentido, las reformas de los planes de estudio de educación cívica (2008) y de estudios sociales (2016) se realizaron en el contexto de la homogenización del sistema educativo costarricense, con base en el modelo por habilidades, cuya premisa es que existen habilidades cognitivas universales que todo sujeto debe desarrollar, según las exigencias de la ciudadanía planetaria y el mercado laboral.

1 En Costa Rica desde 2008 se aplican las "Pruebas nacionales diagnósticas de Segundo Ciclo" (PND) y a nivel internacional las del "Tercer estudio regional comparativo y explicativo" (TERCE), diseñadas por el Laboratorio Latinoamericano de Evaluación de la Calidad de la Educación (LLECE), de la UNESCO. En 2009 se empezó a participar de las pruebas del Programa Internacional para la Evaluación de Estudiantes (PISA, por su sigla en inglés). 
Se aclara que no se abordarán cuestiones metodológicas, sino elementos propiamente teóricos relativos a la relación entre pensamiento y saber social, ciudadanía y el papel de la enseñanza de las ciencias sociales en el marco de la hegemonía del discurso de la calidad educativa y su relación con el capitalismo contemporáneo. Las limitaciones del artículo son consecuencia de esta propuesta analítica propiamente documental, cuyas fuentes son la documentación oficial de la política educativa, los fundamentos curriculares y el plan de estudios sociales para secundaria. Lo anterior se complementó con la revisión de informes de organizaciones internacionales involucradas en los procesos de transformación educativa inscritos en el discurso de la calidad educativa, los cuales contribuyen a desvelar la imbricación entre la asignatura y el discurso de la calidad educativa.

En cuanto al marco teórico del escrito, este parte de los trabajos de Sebastián Plá (2014; 2018), particularmente en la forma como el autor establece las relaciones entre el discurso de la calidad educativa y los conceptos de educación escolarizada y pensamiento social. El primero ya introducidos párrafos atrás. Sobre los dos últimos conceptos mencionados, se parte de una concepción de la educación escolarizada como espacio de interacción social y cultural, interpelado por elementos políticos articulados desde el currículo y la política educativa, por un lado; por otro, se entiende el saber social como un tipo de conocimiento cuya base son las ciencias sociales, el que permite conocer y actuar sobre la sociedad; entonces, el aprendizaje de lo social se fundamenta (como objetivo y aspiración) en el desarrollo del pensamiento y la consecuente acción de los sujetos como producto de la capacidad de comprender su entorno y así mismos.

Las acepciones anteriores entran en disonancia con lo planteado en las reformas educativas acaecidas en Costa Rica desde 2008. Ello provoca una serie desplazamientos epistémicos conceptuales, los cuales no solo refieren al proceso de selección de los contenidos o temáticas que conforman el currículo de la asignatura, sino a la resignificación de la triada esencial de la asignatura: el sujeto ciudadano, el pensamiento y los saberes sociales. Entender estos desplazamientos supone un reto 
esencial para generar caminos alternos que replanteen, con una sólida base epistémica y política, el objeto de la enseñanza de las ciencias sociales en clave crítica.

Por otra parte, el artículo se estructura en tres apartados y dos subapartados. El primero plantea una descripción puntual de la reforma educativa de la política y el currículo nacional, y los elementos que trazan vínculos con el discurso de la calidad educativa. Seguidamente, se identifica la manera en que el aprendizaje y las habilidades, definidas desde la calidad educativa, estructuran el currículo nacional y establecen los parámetros del saber e, incluso, de la enseñanza. Por último, se revisa el cómo las habilidades se insertaron en el programa de estudios sociales, en un juego discursivo que intenta encontrar un balance entre las habilidades genéricas derivadas de la ciudadanía planetaria y la pretensión del desarrollo de pensamiento histórico y geográfico. Finalmente se sintetizan las ideas expuestas en el texto en varios puntos a modo de conclusión.

\section{Reforma educativa costarricense y calidad educativa}

Al menos desde inicios del siglo XXI existía la preocupación de reformar la política educativa en Costa Rica. Para ello se estableció una comisión que trabajaría en la creación de una política guiada por los siguientes ejes: “...calidad, pertinencia y equidad de la educación; evaluación de la educación costarricense; gestión eficiente de la educación; recursos humanos del sistema educativo; empoderamiento de la comunidad educativa; y marco legal y regulatorio de la educación costarricense" (MEP, 2008, p. 2).

La siguiente administración, al mando de Leonardo Garnier (20062010), asumió el "Acuerdo nacional por la educación”. El resultado fue la política "El centro educativo de calidad como eje de la educación costarricense" (2008). Esta propuesta no derogó la política educativa para el siglo XXI de 1994. Más bien sirvió de base teórica para consagrar los tres ejes sobre la naturaleza de la educación: racionalismo, constructivismo y humanismo. Ya con el primer gobierno del Partido 
Acción Ciudadana y su ministra de educación Sonia Marta $\mathrm{Mora}^{2}$, se aprobó, según acuerdo n. ${ }^{\circ}$ 07-64-2016 del 17 de noviembre de 2016, la política educativa "La persona: centro del proceso educativo y sujeto transformador de la sociedad".

Los datos no deben tomarse como algo baladí. Indican la construcción compleja, y a veces contradictoria, sobre la que se intenta erigir la escuela costarricense del siglo XXI dentro del discurso hegemónico de la calidad educativa. El papel de organismos como la OCDE, el Banco Mundial y principalmente el informe del Estado de la Educación adscrito al CONARE ha sido determinante para que la relación entre desarrollo, equidad y educación sea asumida como verdad incuestionable.

Así mismo, en el informe sobre la situación de la educación costarricense de 2017, la OCDE estableció pautas urgentes por modificar y también validó las reformas realizadas en la última década. Ello fue secundado por los estudios del Estado de la Educación $(2017 ; 2019)$ que llaman a atender lo que el BM llamó "crisis del aprendizaje". Las acciones sugeridas buscan el giro de la educación hacia la promoción de las habilidades del estudiantado en el proceso de escolarización frente a los pobres resultados de las pruebas PISA.

La política vigente se constituyó sobre los principales referentes del discurso de la calidad educativa y la establece en los siguientes términos:

...principio nuclear que articula otros principios clave como la inclusión y equidad, el respeto a la diversidad, la multiculturalidad y pluriculturalidad, la igualdad de género, la sostenibilidad, la resiliencia y la solidaridad, así como las metas educativas que fomentan la formación humana para la vida, con el desarrollo de habilidades, destrezas, competencias, actitudes y valores (Ministerio de Educación Pública, 2017, p. 10). ${ }^{3}$

2 Fue además rectora de la Universidad Nacional de Costa Rica entre 2000-2005, periodo en el cual se trimestralizó el ciclo educativo universitario. Además, fue presidenta (2013) del Sistema Nacional de Acreditación de la Educación Superior, institución creada en 2002 para establecer parámetros sobre la calidad de la educación superior. No en balde la reforma más fuerte bajo los criterios de eficiencia y eficacia y enfoque de habilidades se dio en su administración como ministra de educación.

3 El énfasis es propio. 
Es de resaltar cómo el concepto de calidad no se limita estrictamente a cuestiones pedagógicas, sino que implica un sine qua non para engarzar el desarrollo económico, el bienestar social y la inclusión. Ello coloca el concepto en una posición de legitimidad total e incuestionable, pues obviarlo implicaría retrasar el desarrollo del país y aumentar la desigualdad. La educación desde esta lectura es más que un motor de movilidad social, es la posibilidad de establecer una base social competitiva a nivel mundial que genere estabilidad social y política (Programa Estado de la Nación en Desarrollo Humano Sostenible, 2019, p. 20).

La idea anterior no es nueva, ya que el énfasis en el aprendizaje tiene antecedentes lejanos, específicamente en la idea de aprender a aprender que, como afirma Plá (2018), desde la década de 1970 impregna el discurso pedagógico de la UNESCO. Con las teorías del capital humano, el concepto de aprendizaje, al lado de la calidad, adquiere un tono completamente económico. Las palabras de Jim Yong Kim, presidente del BM, definen diáfanamente esto al afirmar: "Lo que verdaderamente importa y genera rentabilidad es aprender y adquirir habilidades. Esto es lo que en realidad crea capital humano" (Banco Mundial, 2018, p. v).

Por consiguiente, el aprendizaje desde la visión del BM tiende a desplazar la atención sobre la naturaleza del conocimiento, al centrarse en lo procedimental enfocado en el logro de indicadores:

Los procesos educativos de calidad privilegiarán la centralidad del aprendizaje de la persona estudiante con el fin de asegurar competencias que propicien la comprensión, expresión e interpretación de conceptos, pensamientos, sentimientos, hechos y opiniones, para permitirle a la persona estudiante interactuar en forma provechosa en todos los contextos posibles (Ministerio de Educación Pública, 2017, p. 12) ${ }^{4}$.

Según la cita, se infiere sintonía entre lo planteado por el BM debido a que se persigue disponer de objetivos formales de aprendizaje medibles $\mathrm{y}$, por ende, evaluables. A pesar de lo evidente, nótese que la estrategia discursiva emplea adjetivos relativos a procesos cognitivos y no cognitivos, revistiendo de nociones humanistas el proceso de

$4 \quad$ El énfasis es propio. 
aprendizaje al aludir a la importancia del desarrollo de esas habilidades en la dinámica social de los individuos.

En suma, la somera descripción realizada sobre la reforma educativa costarricense realizada en este apartado evidencia la estrecha relación con los postulados de la calidad educativa determinados tanto por la UNESCO, como por el BM y la OCDE. Los matices y particularidades de la implementación de este planteamiento adquieren mayor claridad al revisar el currículo nacional, que a fin de cuentas es el instrumento de operacionalización de los postulados. El currículo basado en el aprendizaje y desarrollo de habilidades incide en la concepción del aprendizaje, de la ciudadanía y del conocimiento que constituyen el marco referencial del programa de estudios de la asignatura. No obstante, surge la cuestión: ¿qué son las habilidades y cómo se presentan en el currículo educativo costarricense? Es lo que trata el siguiente apartado.

\section{Aprendizaje y desarrollo de habilidades}

Como se planteó, la política educativa nacional abrazó los principios de la calidad educativa. La operacionalización se encuentra detallada en el currículo nacional. El núcleo del currículo es la primacía del aprendizaje y su relación con el desarrollo de habilidades. Esto constituye un desplazamiento epistémico pedagógico que no puede ser trivializado. Ello, porque la centralidad del aprendizaje no es el problema, sino la relación entre aprendizaje y habilidades en función de la demanda del mercado laboral, por un lado; por otra parte, la universalidad de las habilidades y el carácter mesurable que las hace medibles y comparables a nivel global a través de un listado de indicadores de aprendizajes por lograr y la noción de ciudadanía planetaria.

Dicho eso, ¿en qué consiste un currículo basado en habilidades? Mauricio Portillo, funcionario del Departamento Dirección Curricular del MEP, sintetiza la clasificación de las habilidades que debe desarrollar un sistema educativo de calidad, según la OCDE:

...el uso interactivo de herramientas (uso del lenguaje, los símbolos y los textos, uso interactivo del conocimiento y la información; uso 
interactivo de la tecnología), la interacción con grupos heterogéneos (relacionarse bien con otras personas, manejar y resolver conflictos, cooperar y trabajar en equipo,) y la acción autónoma (actuar dentro del contexto del gran panorama, formar y conducir planes de vida, defender y asegurar derechos) (2017, párr. 15).

Si se observa con calma, es evidente el paralelismo entre esos postulados y los pilares de la política educativa. Las habilidades adquieren, entonces, carácter total en parte por la necesidad de ajustar lo que la escuela produce y lo que el mercado laboral requiere. Las habilidades son totales porque se presentan como aquello que es necesario para la sociedad del conocimiento. Estas habilidades, siguiendo a Plá, constituyen la supracognición. Esta se define así:

...es una categoría política no cognitiva. No hace referencia a los procesos cognitivos en sí, sino que su intención es fijar la atención en las relaciones de poder entre conocimientos que establecen jerarquías a partir de lo que se define como naturaleza del aprendizaje, que no es otra que las habilidades y competencias exigidas por la sociedad del conocimiento (2018, p. 25).

Según esta interpretación, la racionalidad propia del discurso de la calidad educativa ha convertido la dimensión política de la supracognición en un problema pedagógico, en tanto su relación con habilidades disloca la relación entre pensamiento producido en la escuela y se enfoca en la operativización e instrumentalización del pensamiento y la conducta del estudiantado. Este ya no es sobre el qué sino sobre el cómo gestionar información de forma "crítica y sistemáticamente" ante posibles situaciones. Es el know how tan valorado por el mundo empresarial. En consonancia con esto, el currículo propone articular el aprendizaje en cuatro dimensiones, a saber:

Formas de pensar: se refiere al desarrollo cognitivo de cada persona, por lo que implica las habilidades relacionadas con la generación de conocimiento, la resolución de problemas, la creatividad y la innovación. Formas de vivir en el mundo: es la dimensión que conlleva el desarrollo 
sociocultural, las interrelaciones que se tejen en la ciudadanía global con el arraigo pluricultural y la construcción de los proyectos de vida. Formas de relacionarse con otros: se relaciona con el desarrollo de puentes que se tienden mediante la comunicación y lo colaborativo. Herramientas para integrarse al mundo: es la apropiación de las tecnologías digitales y otras formas de integración, así como la atención que debe prestarse al manejo de la información (Ministerio de Educación Pública, 2015, p. 25).

Cada dimensión cuenta con una batería de indicadores y aprendizajes por lograr, que sirven de parámetros mesurables del desarrollo o no de las habilidades inherentes al sujeto ciudadano planetario. Lo que sucede es que la retórica del currículo, si bien no oculta que las habilidades son parte del correlato del capital humano, las combina con postulados que conceptualmente enlazan con principios de la escuela nueva y la pedagogía crítica, creando una falsa identidad crítica y humanista sobre las dimensiones de aprendizaje. Ejemplo de lo afirmado, nótese que en la dimensión "formas de pensar" los indicadores están categorizados en: pensamiento crítico, pensamiento sistémico, aprender a aprender, resolución de problemas y creatividad e innovación (Ministerio de Educación Pública, 2015, p. 26), las cuales articulan las "formas de vivir en el mundo" donde la habilidad se centra en actitudes desde la noción de ciudadanía planetaria y el desarrollo de creatividad para "producir". La condición "crítica" no remite a la tradición crítica epistemológica sobre el sujeto y el saber de la escuela de Frankfurt o a Paulo Freire.

Por el contrario, el planteamiento curricular establece la retórica descrita, mientras que operativamente establece los límites de las habilidades cognitivas dentro del aprendizaje y su dislocación del pensamiento como proceso más profundo en la constitución de la subjetividad del estudiantado. De tal manera, la habilidad se estructura en función de la posibilidad de que produzca aprendizajes medibles a nivel mundial. En este punto, ya se revela que el aprendizaje medible de la forma de pensar (crítica o sistémica) es solamente la gestión y uso del dato de determinadas temáticas según las asignaturas, cuya ilusión es que esto implica pensamiento científico. 
En definitiva, el aprendizaje como categoría central inscrito en el marco de la supracognición "trata de formar en un pensamiento genérico y flexible que permita la creación de una base laboral lo suficientemente amplia para la reproducción del capitalismo actual" (Plá, 2018, p. 397). Su alcance es superficial en tanto logre garantizar el aumento de los resultados de las pruebas estandarizadas y dé señales, al mercado laboral, de que la inversión educativa garantiza los réditos, al generar la oferta de trabajadores y trabajadoras.

Por ejemplo, el VII informe del Estado de la Educación hace hincapié en que los niveles de inversión educativa son muy elevados para los resultados tan pobres:

Recursos no sobran; simplemente no están bien asignados en función de las grandes prioridades que el sistema debe resolver y que el país requiere. Podría hacerse más con los actuales niveles de inversión y, además, es preciso efectuar importantes cambios en los destinos y en la transparencia de esa inversión, pero eso no significa que esta sea suficiente para sufragar una mejor educación (2019, p. 18).

Es importante reconocer que, como indica la cita, desde este informe no se pretende una disminución del presupuesto destinado a la educación, sino una política de gestión educativa que sea eficiente, bajo los parámetros de la calidad educativa. El papel de un informe de esta naturaleza reside en que otorga legitimidad a las reformas por realizar o realizadas mediante evidencia empírica, un velo de objetividad científica que elimina, de la discusión, su dimensión política y cultural. En este punto, el aprendizaje nada tiene que ver ni con el principio socioconstructivista ni humanista de la política educativa vigente, principalmente porque aprender tiene que ver más con la eficiencia de los procesos de enseñanza que con el pensamiento del estudiantado.

No es casualidad que esta forma de entender el aprendizaje implique otro desplazamiento conceptual, esta vez sobre la función docente. En el informe sobre las políticas públicas docentes ya se habla de "enseñanza efectiva”, categoría que: “...explora si existen los elementos preparatorios fundamentales para permitir una instrucción de calidad tales 
como expectativas claras, tiempo de clase adecuado y preparación docente sólida" (Programa Estado de la Nación-BID, 2018, p. 7). ${ }^{5}$ Los tres criterios de la "enseñanza efectiva" son producto de las investigaciones del Estado de la Educación en áreas específicas y congruentes con las pruebas estandarizadas aplicadas en el país. Las áreas son lecto escritura, matemáticas y ciencias.

Según la evidencia que sustenta la categoría, el profesorado costarricense, desde preescolar hasta secundaria, presenta serios problemas para cumplir con los indicadores de aprendizaje de los programas de estudio que han sido reformados desde 2009. La deficiencia tiene que ver con que la formación universitaria no basa el currículo en lo que debe enseñar cada asignatura escolar, no se tienen conocimientos metodológicos actualizados o carecen de una didáctica acorde con el enfoque de habilidades y persiste la enseñanza de contenidos (Programa Estado de la Nación-BID, 2018).

Todas estas afirmaciones, ya convertidas en criterios científicos objetivos, denotan, en el fondo, la tecnificación de la enseñanza. ¿Por qué si los indicadores poseen una dimensión universal para determinar la calidad del aprendizaje la enseñanza habría de operar desde otros criterios pedagógicos? La estandarización también se aplica a la forma de enseñar que convierte el plan de estudios en un objeto inamovible, lo que guarda una contradicción en los principios filosóficos de la política educativa que pretende el respeto a la diversidad de pensamientos. Lo otro, además, es la forma grosera de obviar la condición intelectual de quien enseña, y cómo su subjetividad incide en la forma que concibe el aprendizaje.

Desde hace tiempo investigadores como John Elliot (1994), William F. Pinar (2015) y Michael F. Young (2010) dan cuenta de la relación entre docentes y currículo, coincidiendo en que la interpretación docente del currículo genera múltiples formas de enseñar marcadas por el acento que le otorga quien enseña a temas, recursos y estrategias didácticas. Para la visión defendida por el Estado de la Educación, el profesorado tiene que ser muy competente para aplicar correctamente el currículo y los planes de estudio; su habilidad pedagógica se mide por el logro de los indicadores.

$5 \quad$ El énfasis es propio. 
Es un despojamiento de la capacidad profesional e intelectual docente, pues su función está hipertecnificada: enseña efectivamente para el logro específico de las habilidades curriculares, ni más ni menos.

\section{El lugar de los estudios sociales: Ciudadanía planetaria, tiempo y espacio histórico}

En el contexto de una enseñanza hipertecnificada y la reforma educativa en el marco del modelo de habilidades, ¿qué lugar ocupan los estudios sociales? En este apartado se propone un recorrido del concepto y su contexto intelectual hasta desembocar en el análisis del plan de estudios de la asignatura en el contexto de la reforma educativa descrita en los apartados anteriores.

En primer lugar, la enseñanza de las ciencias sociales, social studies o estudios sociales, como asignatura escolar en Iberoamérica (Pagés, 2009) y el mundo anglosajón (Evans, 2006) ha logrado subsistir, no sin polémicas y dificultades, con un objetivo muy concreto: formar la ciudadanía en el modelo democrático republicano occidental a través de la enseñanza de saberes disciplinares como la historia, geografía, cívica o política. Ello ha mantenido a la asignatura en movimiento entre dos posiciones, que el profesor Joan Pagés sintetizaba de la siguiente manera:

Parece que a principios del siglo XXI subsisten estos dos enfoques en cuanto a las finalidades de la enseñanza de las ciencias sociales: por un lado, las que están destinadas a proporcionar a las nuevas generaciones una idea de aquello que es permanente en la vida de una nación y, por el otro, las que apuestan por enseñar a criticar la tradición que se transmite a través de los estudios sociales $(2009$, p. 6$)$.

Estos enfoques mencionados han trazado cuestiones relevantes y han movilizado la construcción de un área de conocimiento específico: la didáctica de las ciencias sociales y los social studies. La abundante investigación de las últimas tres décadas muestra nuevas líneas temáticas en torno a la enseñanza de las ciencias sociales: el cómo enseñar y aprender historia, las características del pensamiento histórico que supone se 
producen desde la asignatura, en si debe primar el conocimiento disciplinar en los contenidos o más bien problemáticas sociales; técnicas y estrategias didácticas según el impacto de las tecnologías de la comunicación, los enfoques de género y la teoría queer, la decolonización, entre otros. Son algunos ejemplos del piso epistemológico propio de la didáctica de las ciencias sociales (Lestivk y Tyson, 2008; Pagés y Santisteban, 2011) y donde los estudios sociales se han nutrido directa o indirectamente.

Empero, ambos enfoques contienen, en su núcleo, la relación entre el pensamiento social e histórico y la noción de ciudadanía que desde la asignatura se debe promover y desarrollar. Esta constante, vista desde el prisma de la reforma educativa y curricular descrita en los apartados anteriores, provoca dudas sobre qué saber social e histórico promueve el programa de estudios y cuál es su relación con las habilidades. En definitiva, ¿cuál es el lugar y función de la asignatura en el modelo educativo por habilidades? Antes de entrar conviene contextualizar el itinerario del programa de estudios sociales entre 2014 y 2016.

\section{a. El itinerario del programa de estudios de la asignatura: Un resumen $^{6}$}

Antes de finalizar sus funciones como ministro de educación Leonardo Garnier, se aprobó intempestivamente un plan de estudios para la asignatura que se asemejaba mucho en sus estructura y contenido al programa Ética, Estética y Ciudadanía. Desde 2012 se venía trabajando en ello, y se publicaron dos versiones, una en noviembre de 2013 y la siguiente en marzo de 2014. Las críticas llegaron de todas partes: gremio sindical y las unidades académicas de las universidades públicas que emitieron criterios desfavorables al programa. La oposición desembocó en la derogación de dicho plan en mayo de 2014 y el llamado de la nueva ministra, en ese momento Sonia Marta Mora, para la conformación de

6 El asesor nacional de estudios sociales, Marvin Carvajal, en su blog ha realizado una encomiable labor de recopilación de todo el material sobre los planes de estudios sociales entre 2012 y 2017. Documentos oficiales y de los miembros de la comisión mixta se pueden consultar en extenso en el blog: http://geografiahistoriasecundaria.blogspot.com/p/blogpage_ $85 . h$ tml 
una comisión mixta de especialistas de las universidades estatales que imparten la carrera de enseñanza de los estudios sociales.

Entre mediados de 2014 y agosto de 2015, la comisión mixta produjo buena cantidad de insumos de muy variado enfoque teórico. Pero en la circular DVM-AC-1145-09-2015 con fecha del 24 de setiembre de 2015, la exviceministra académica comunicaba al director del Departamento de Desarrollo Curricular del MEP que el Consejo Superior de Educación (CSE) daba por terminada la participación de la comisión mixta. En el acuerdo 06-51 del CSE no hay explicación alguna de las razones para dicha decisión, más acuciante aún, nada se dice sobre la versión propuesta por la comisión mixta para los programas de III ciclo y diversificada.

El programa propuesto por la comisión con fecha del 20 de julio de 2015 propuso un giro disciplinar, donde el aprendizaje de la historia y la geografía se ofrecía más detalladamente en cuanto a la promoción de cualidades o habilidades propias de ambas disciplinas: tiempo histórico, manejo de fuentes, pensamiento espacial, entre otras. Las temáticas resultaron más sugerentes, como, por ejemplo, el desarrollo del poder en las sociedades en perspectiva histórica y la relación entre uso de espacios y capitalismo (pp. 74-78). ¿Era una mejor propuesta para la asignatura que la actual?, pregunta engañosa y complicada, debido a la diversidad de enfoques y formas de comprender los estudios sociales.

Me interesa señalar que la principal diferencia de la propuesta de la comisión y el programa actual es que el primero planteaba las habilidades organizadas en tres dimensiones: cognitivas, procedimentales y socioculturales, manteniendo cierta distancia con el modelo de habilidades de la calidad educativa ya descrito páginas atrás. Otra diferencia es que las temáticas propuestas como vehículos de conocimiento respondían al desarrollo de habilidades propias de historia y de la geografía, y el enfoque sociocultural pretendía enmarcar el saber social con la experiencia ciudadana del estudiantado. Al menos, del escrito se puede inferir que esa era la pretensión de la comisión. Con la disolución de la comisión, la propuesta fue, en buena parte, desechada. Quedó solamente la gran experiencia del debate interuniversitario y gremial. Mas 
allá de ello, el resultado fue el programa de estudio que empezó a regir oficialmente en 2017. El siguiente subapartado realiza un análisis sobre la relación entre las habilidades y la particularidad del saber social que propone el programa.

\section{b. Ciudadanía planetaria y saber social}

A nivel internacional no existen habilidades específicas evaluables sobre la enseñanza de las ciencias sociales, la historia, geografía o la cívica. Ya he dicho que son tres las áreas: lecto-escritura, ciencias y razonamiento matemático. ¿Para qué los estudios sociales, entonces? Entre la justificación epistemológica que propone el programa de estudios y el currículo nacional (2016; 2015), se establece que la educación es motor de bienestar social, de la persona y del colectivo. Para lo cual se requiere formar en una nueva ciudadanía, es decir, una ciudadanía propia del mundo globalizado:

La nueva ciudadanía procura insertarnos en la ciudadanía planetaria con identidad nacional lo cual significa fortalecer y reconocer nuestra memoria histórica, para ser conscientes de quiénes somos, de dónde venimos y hacia dónde queremos ir. En ese sentido debemos recordar que un pueblo que pierde su memoria histórica integrada erróneamente comienza a mirar su historia y su cultura como hechos estáticos, aislados e inconexos, ocurridos en un tiempo pasado lineal que vino y se fue, sin que tengamos nada que recuperar de esos hechos que se recuerdan sin responder a las preguntas del por qué y el cómo ocurrieron, sin aportar ninguna enseñanza para las actuales y futuras generaciones. Por tal motivo, el fortalecimiento de nuestra identidad nacional es fundamental para elegir caminos idóneos para integrarnos, de la mejor manera posible, a una ciudadanía planetaria, con la plena conciencia de que ello contribuye al progreso individual y social (Ministerio de Educación Pública, 2015, p. 21) ${ }^{7}$.

$7 \quad$ El énfasis es propio. 
Con este parámetro, el programa postula la categoría de estudiantado como ciudadano global o planetario y se justifica de la siguiente forma:

El desarrollo de un nuevo programa de Estudios Sociales es un aporte fundamental a la construcción de esa nueva ciudadanía pues desde aquí se abordan perspectivas, enfoques y habilidades geográficas e históricas, que permiten oportunidades al estudiantado para comprender, explicar y actuar en la sociedad compleja, dinámica y retadora que vivimos. A partir del desarrollo del pensamiento crítico y por medio de un aprendizaje socioconstructivista enfocado en la resolución de problemáticas, se procura potenciar las oportunidades que el estudiante tendrá para su desarrollo de un ser pleno y activo de la sociedad nacional y ejercer también con responsabilidad su ciudadanía planetaria (Ministerio de Educación Pública, 2016, p. 8).

Ambos documentos, con el lenguaje neoestructuralista de la CEPAL (CEPAL, 1990), proponen que una condición para superar la desigualdad es el aprovechamiento de las oportunidades, donde la educación es una de ellas y el aprendizaje de los estudios sociales un saber valioso para lograr el objetivo. Esa posición podría resumirse así: el Estado se encarga de generar oportunidades, pero es el sujeto quien, al aprovechar la educación diseñada para que adquiera capacidades de desarrollo, responsable de su bienestar (Leiva, 2008).

La categoría de ciudadanía global sirve de eslabón para sugerir la necesidad de ciertas habilidades que requiere el sujeto para alcanzar el desarrollo. En consecuencia, la tabla 1 muestra el entramado que propone el currículo nacional en la dimensión 1 con la habilidad del pensamiento sistémico en cada nivel escolar. La propuesta denota gradualidad en cuanto al desarrollo del pensamiento sistémico en dos elementos: tiempo y espacio histórico, y manejo de datos. Se inicia con la localización, que alude a cierta concreción conceptual acorde al nivel, y en la medida que pasa de nivel las operaciones no dejan de ser estrictamente procedimentales en cuanto al manejo de información. 


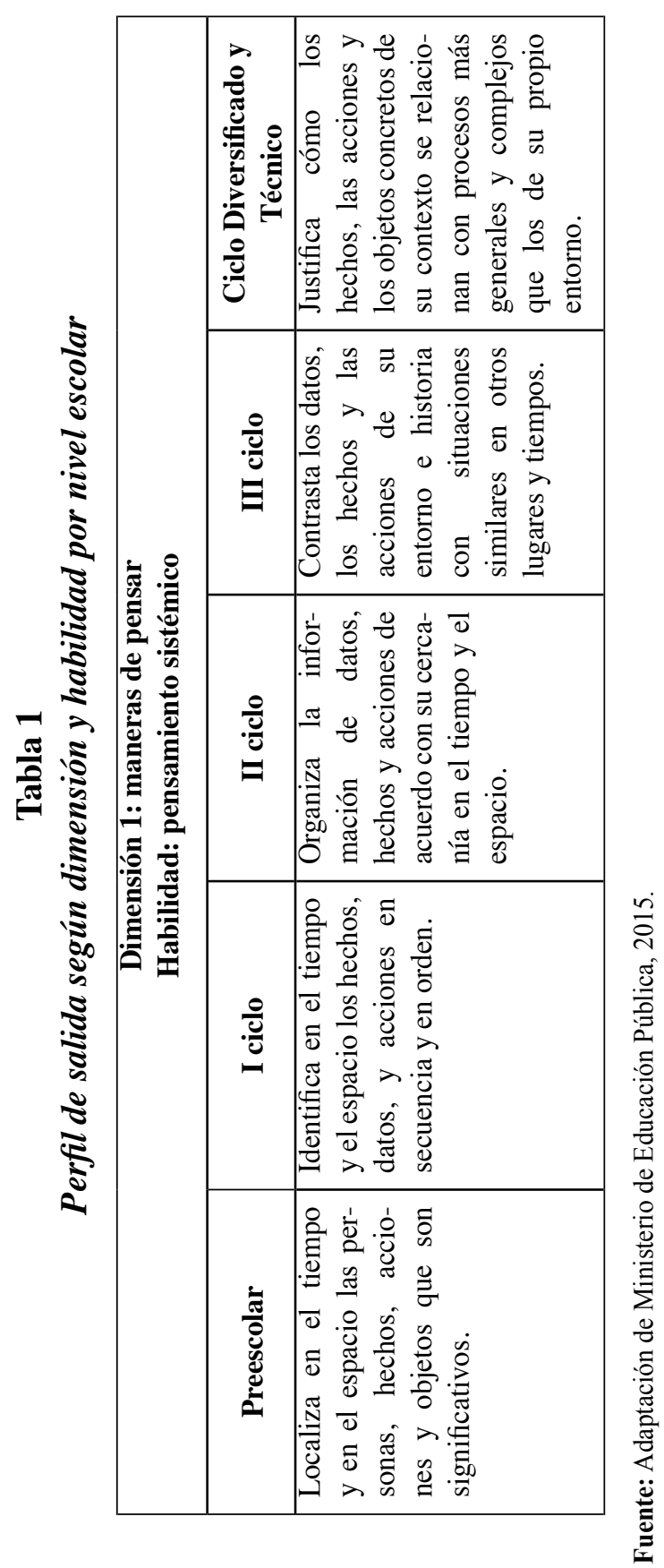


Tanto la dimensión como la habilidad de pensamiento sistémico están diseñadas para el ciudadano global o planetario, el cual construye su cotidianidad más allá de los límites de la comunidad nacional. El programa de estudios de la asignatura, en este marco totalizante, parte de la premisa de que desde los estudios sociales es posible generar capacidades derivadas del pensamiento histórico y geográfico, que permitan "pensar globalmente y actuar localmente". Con esto admite que el conocimiento y las capacidades, necesarias para el bienestar, se establecen y son universales. El carácter supracognitivo se manifiesta en este primer nivel.

En cuando al perfil de salida del programa en la Tabla 2, los indicadores revelan dos categorías operativas del aprendizaje: tiempo y espacio histórico geográfico.

\section{Tabla 2}

\section{Perfil de salida de estudios sociales tercer ciclo}

\begin{tabular}{|l|l|}
\hline \multicolumn{2}{|c|}{ Dimensión I: maneras de pensar } \\
\hline Habilidad & Indicadores \\
\hline \multirow{4}{*}{$\begin{array}{l}\text { Pensamiento } \\
\text { sistémico }\end{array}$} & $\begin{array}{l}\text { Contrasta los datos, los procesos, los hechos y las acciones de } \\
\text { su entorno e historia personal con situaciones similares en otros } \\
\text { lugares y otros tiempos. }\end{array}$ \\
\cline { 2 - 3 } Pensamiento & $\begin{array}{l}\text { Interrelaciona diferentes tipos de información geográfica e his- } \\
\text { tórica sobre un tema. }\end{array}$ \\
\cline { 2 - 3 } crítico & $\begin{array}{l}\text { Incorpora conocimientos, técnicas y herramientas geográficas } \\
\text { e históricas para comprender su entorno. }\end{array}$ \\
\hline \multirow{4}{*}{$\begin{array}{l}\text { Verifica que los datos o ejemplos utilizados en el estudio de la } \\
\text { Historia y la Geografía sean precisos, para ser utilizados en un } \\
\text { razonamiento. }\end{array}$} & $\begin{array}{l}\text { Interpreta en forma precisa en su cultura cotidiana, conceptos, } \\
\text { mapas, gráficas, símbolos, signos y preguntas, entre otras for- } \\
\text { mas de expresión de la aplicación de los Estudios Sociales. }\end{array}$ \\
\cline { 2 - 3 } & $\begin{array}{l}\text { Profundiza en el significado de conceptos claves de la Geogra- } \\
\text { fía y de la Historia para el entendimiento de una argumentación. }\end{array}$ \\
\hline
\end{tabular}




\begin{tabular}{|c|c|}
\hline \multicolumn{2}{|r|}{ Dimensión I: maneras de pensar } \\
\hline Habilidad & Indicadores \\
\hline \multirow{3}{*}{$\begin{array}{l}\text { Aprender a } \\
\text { aprender }\end{array}$} & $\begin{array}{l}\text { Aprovecha las oportunidades de aprendizaje disponibles, los } \\
\text { obstáculos y sus habilidades personales para enriquecer y } \\
\text { expresar conocimiento histórico y geográfico. }\end{array}$ \\
\hline & $\begin{array}{l}\text { Revisa que las estrategias de trabajo elegidas sean efectivas de } \\
\text { acuerdo con las expectativas. }\end{array}$ \\
\hline & $\begin{array}{l}\text { Acepta la realimentación con otras personas para mejorar el } \\
\text { trabajo individual y grupal. }\end{array}$ \\
\hline \multirow{2}{*}{$\begin{array}{l}\text { Resolución de } \\
\text { problemas }\end{array}$} & $\begin{array}{l}\text { Analiza un problema a partir de lo conocido y de la necesidad } \\
\text { de información geográfica e histórica detectada. }\end{array}$ \\
\hline & $\begin{array}{l}\text { Organiza de forma conveniente, los conocimientos geográficos } \\
\text { e históricos, los recursos y las actividades para una solución }\end{array}$ \\
\hline $\begin{array}{l}\text { Creatividad e } \\
\text { innovación }\end{array}$ & $\begin{array}{l}\text { Compara la calidad de sus ideas y las de otras personas de } \\
\text { acuerdo con su novedad y utilidad para el logro de una meta. }\end{array}$ \\
\hline
\end{tabular}

Fuente: Ministerio de Educación Pública, 2016, p. 60.

Por esta razón, las habilidades se justifican "científicamente" desde las disciplinas estructurantes de la asignatura: la historia y la geografía. Estas ofrecen herramientas y conocimientos necesarios para ciertos procesos.

... el desarrollo de pensamiento crítico, resolución de problemas, habilidades de indagación recolección-análisis de datos e información, colaboración, para evaluar y tomar posición sobre diversos recursos y temas, así como reconocer las diferencias entre el interés individual y el colectivo. Por otra parte, también significa el desarrollo de conocimiento en diversas disciplinas de las ciencias sociales, en particular la Historia y la Geografía, con el fin de comprender los diferentes elementos que inciden o afectan la sociedad pasada, presente y futuro (2016, p. 17).

Hay que advertir que el pensamiento histórico es una categoría, tanto de la didáctica de la historia y las ciencias sociales, como de la teoría histórica. No es una categoría monolítica y total, pues la bibliografía especializada distingue enfoques sobre el pensamiento histórico, por ejemplo, los que asumen posturas desde la filosofía histórica sobre 
la conciencia histórica de Jorn Rusen, como Peter Seixas y Stephane Levesque; los de corte sociocultural, entre ellos Keith Barton y, el más difundido, el enfoque cognitivista, cuyo autor más representativo es Mario Carretero.

No pretendo señalar que un enfoque teórico es mejor o peor que otro, no me interesa una discusión de ese tipo en la medida en que cada posición epistemológica ha vislumbrado elementos valiosos para la enseñanza de la historia. El problema es que, como indica Plá para el caso mexicano y en particular de la enseñanza de la historia:

El tiempo y el espacio históricos como noción de aprendizaje son creaciones didácticas y psicológicas, pero no historiográficas. El objeto de investigación creado por los historiadores no es el tiempo o en su caso el espacio, sino el ser humano en sociedad en un espacio y en un tiempo determinado. Sin embargo, en la necesidad -primero de los psicólogos constructivistas de buscar la especificidad del pensamiento histórico en un sujeto cognoscente universal y después de las políticas educativas para medir la calidad educativa a partir de resultados- por encontrar un esquema válido de pensar el pasado, determinaron que el tiempo y el espacio históricos son competencias cognitivas. ... las propuestas para la enseñanza de la historia atraen desde la psicología la noción de sujeto cognoscente universal y lo convierten en objeto mensurable y por tanto objeto de enseñanza, arguyendo proximidad con el saber discipli$\operatorname{nar}(2014)$.

Al extrapolar a los estudios sociales esa aseveración, claramente la apuesta del programa es realizar una conexión entre habilidades, ciudadanía planetaria y disciplina histórica y geográfica. Aquí entra la cuestión del conocimiento y el pensamiento. Dotar de peso científico el desarrollo de habilidades del pensamiento histórico justifica la asignatura en el marco del modelo curricular, pues la hace "un saber valioso" y relevante:

Estudiar Historia dentro de los Estudios Sociales implica, en ese sentido, el desarrollo de una sensibilidad para poder ubicar históricamente (en tiempo) las formas de desarrollo, continuidad y transformación de la humanidad. La Historia le brinda además a los estudiantes un conjunto 
de herramientas que les permitirá desarrollar destrezas para entender la política, la economía, la sociedad y la cultura y les ayudará a profundizar en el uso de textos históricos para construir argumentos que evalúen el presente y el futuro. En resumen, la enseñanza de la Historia permite crear ciudadanos conocedores de su pasado, que pueden ubicar procesos en el tiempo, que saben leer textos históricos y que tienen la capacidad de determinar en su sociedad los efectos del pasado de cara al futuro (Ministerio de Educación Pública, 2016, p. 16).

El problema del saber y el conocer aquí no es poca cosa. El carácter total y universal de las habilidades históricas se inscriben en un pensamiento "crítico" igual de utilitario, pues disloca la posibilidad de preguntar por las fisuras de las problemáticas sociales, en tanto lo que se pretende es la aplicación de estrategias de validación de la realidad. La selección de las temáticas históricas y geográficas se inscriben en aquello que el modelo de fronteras planetarias y los objetivos del desarrollo sostenible consideran conocimiento relevante y válido socialmente (Ministerio de Educación Pública, 2015).

Lo descrito se hace más evidente en sétimo y octavo año, cuyas temáticas son "La ocupación humana y el uso del espacio geográfico: lecciones espaciotemporales del pasado para pensar el presente y el futuro" y "La adaptación del ser humano a un medio cambiante: gestión del riesgo, recursos hídricos y cambio climático", respectivamente. En el primer caso se combina el estudio de las civilizaciones antiguas del mundo y de América, desde la transformación espacial y la modificación del ambiente. En octavo año, la estructura de la tierra y luego el calentamiento global durante dos trimestres. En noveno, décimo y undécimo, se aborda la historia en su periodización más clásica: de la edad media a las sociedades contemporáneas. Se introducen preguntas generadoras que incluyen a las mujeres y grupos subalternos como esclavos y migrantes, todo ello, desde mi punto de vista, enfocado en la identificación de colectivos históricamente marginados, pero no se plantea la problematización sociopolítica de la naturaleza de las diferencias, de la desigualdad ni de la propia producción de formas históricas (Ministerio de Educación Pública, 2016). Se debe enfatizar el hecho de que en el programa no es distinguible la procedencia epistemológica sobre la 
enseñanza de la historia propuesta, se ofrece como una visión unívoca que sintetiza el conocimiento histórico en tanto que no necesariamente se preocupa por el pasado, sino por la constatación desde el presente de procesos, objetos y sujetos históricos.

Conviene en este punto recordar a Max Horkheimer en su crítica a Hegel:

La nebulosidad de ese sujeto universal, al que Kant afirma, pero al que no puede caracterizar satisfactoriamente, es disipada por Hegel en cuanto pone el espíritu absoluto como lo eminentemente real (das Allerrealste). Lo universal, según él, ya se ha desplegado adecuadamente y es idéntico a lo que se concreta. La razón ya no necesita ser simplemente crítica respecto de sí misma; en Hegel ella se ha vuelto afirmativa; aun antes de que la realidad deba ser afirmada como racional (Horkheimer, 2003, p. 237).

A la sazón, lo social en el programa es una realidad producto del pasado, cuyas consecuencias (presente) debemos verificar y, al hacerlo, podremos adquirir un pensamiento sistemático útil para el desarrollo personal en el mundo global. El sujeto ciudadano planetario juega a adaptarse a las dinámicas impuestas por la globalización mediante la implementación de un pensamiento crítico y sistémico que mira "históricamente" y opera como herramienta de verificación de las certezas globales.

Finalmente, el programa supuso avances interesantes al eliminar las listas de contenidos y, con ello, indirectamente, permitir mayor libertad de acción docente; pero esto se disminuye con las claras sugerencias de cómo mediar efectivamente. En este punto, el saber social y el conocimiento histórico propuesto en el programa, muy a pesar de la retórica crítica y las extrañas combinaciones entre postulados de la didáctica de la historia, diametralmente opuestos y aderezados con adjetivos vigotskyanos (Ministerio de Educación Pública, 2016) está supeditado al desarrollo de la habilidad genérica del manejo del tiempo y del espacio. Tal subordinación confirma el ajuste que sufre la asignatura en la reforma educativa tratada. 


\section{Conclusiones}

A lo largo de este ensayo se discutió el impacto de las políticas educativas de calidad y el modelo en habilidades que se ha instalado en Costa Rica en la asignatura de los estudios sociales. Para ello se propuso que la forma para legitimar el modelo por habilidades fue el diseño de una estructura poderosa epistemológica sobre la educación, basada en los principios de rendimiento economicista, desde la cual extrapola conceptos pedagógicos hasta subvertirlos en función de legitimar modelos, currículos y políticas que instalan la razón instrumental como saber escolar. En síntesis, se exponen algunas conclusiones/problemas:

1. Las habilidades pautan y delimitan el saber y la enseñanza "efectiva". A pesar del lenguaje pedagógico progresista utilizado en la política educativa y el programa de estudios, no logra resistir las grietas de un diseño hiperinstrumental que disloca socialmente a docentes y estudiantes de su posibilidad de conciencia sobre sí y para sí; ya que la promoción del pensamiento crítico, complejo y creativo están enfocados en la adquisición de habilidades genéricas que derivan de la historia y la geografía, cuyo uso es la resolución de problemas inmediatos, más que en la comprensión de las interacciones sociales, las dinámicas de poder y la incertidumbre de las sociales de la actualidad.

2. Es importante reconocer que en Costa Rica aun el programa de estudios sociales intenta armonizar los requerimientos del currículo nacional, los postulados de la ciudadanía planetaria y el pensamiento histórico y geográfico, con la finalidad de un conocimiento "valioso" como parte estructurante de habilidades para ejercer la ciudadanía planetaria. El resultado es un cúmulo de enunciados a veces incompatibles y otras profundamente descontextualizados de la investigación en la enseñanza de la historia y la didáctica de las ciencias sociales y social studies.

3. Ello evidencia dos cuestiones centrales. La primera, el programa de estudios de la asignatura vigente nace en la contingencia de la reforma curricular y revela un tanteo para rearticular el papel 
del aprendizaje de los estudios sociales sobre habilidades. En segundo lugar, la obvia exclusión de la asignatura de las pruebas estandarizadas FARO, que da margen tanto a autoridades como a investigadores e investigadoras para profundizar los cambios en el programa de estudio.

4. La asignatura, por tanto, está ciertamente en un espacio indefinido, pues su naturaleza no termina de calzar con el modelo de habilidades. La mantiene a flote el papel que juega para el Estado el uso político del pasado y la historia, a pesar de que en la escuela pueden convivir diversos regímenes de historia.

5. Lo social implica un pensar tanto racional como ontológico y cultural, la subversión a la que se expone en el marco de la supracognición es que opere únicamente de manera referencial del comportamiento desde la sociabilidad del mercado. El saber social en su amplitud, diversidad y complejidad histórico cultural difícilmente puede ser medible si no es por contenidos y temáticas que se refieren a lo procedimental y que extrapolan lo político. El programa juega en este terreno escabroso, donde convierte las categorías tiempo histórico y espacio geográfico en objetos para mediar en el desarrollo de habilidades que trascienden el saber social. No hay opción de construir pensamiento, pues la apuesta es que las habilidades puedan constatar los fenómenos del presente mediante miradas históricas. La realidad se retrotrae a la crítica, pues queda indemne ante la verificación de su objetividad.

6. Los conceptos de enseñanza y el aprendizaje se incluyen en el discurso de la política, descontextualizados de sus lugares de enunciación epistémica y se definen en pos del logro de los indicadores propuestos como medidores de las habilidades. Con ello se obvia la condición subjetiva del ejercicio docente, de la dimensión intelectual que implica seleccionar formas de enseñar, contenidos y la propia reflexión de quien enseña.

7. En el contexto costarricense, la poca investigación en el área de estudios sociales referidas al desarrollo del pensamiento histórico y geográfico no permite establecer debates en igualdad de condiciones 
con las racionalidades de los grupos expertos en habilidades. Ello supone una veta abierta para proponer una agenda de investigación crítica y contribuir en la redefinición de los estudios sociales, en la cual el pensamiento social e histórico logren articular una didáctica con identidad propia, libre de toda disminución tecnificada.

\section{Referencias}

BancoMundial.(2018).Informesobreelsobre el desarrollomundial2018:Aprenderpara hacer realidad la promesa de la educación. doi:10.1596/978-1-4648-1096-1

CEPAL. (1990). Transformación productiva con equidad. CEPAL. http://repositorio. cepal.org/handle/11362/2102

De Sousa Santos, Boaventura. (2006). De la mano de Alicia: Lo social y lo político en la postmodernidad. Siglo del Hombre Editores.

Elliott, John. (1994). The Teacher's Role in Curriculum Development: An Unresolved Issue in English Attempts at Curriculum Reform. Curriculum studies, 2(1), 43-69. http://dx.doi.org/10.1080/0965975940020103.

Fallace, Thomas. (2017). The Intellectual History of the Social Studies Research. En M. McGinn, \& C. Mason (Edits.), The Wiley Handbook of Social Studies Research. John Wiley \& Sons, Inc.

Horkheimer, Max. (2003). Teoría crítica. Amorrourtu.

Jorgensen, C. Georgenssen. (2014). Social Studies Curriculum Migration: Confronting Challenges in the 21 st Century. En E. W. Ross (Ed.), The Social Studies Curriculum. Purposes, Problems, and Possibilities. (pp. 3-24). SunyPress.

Körber, Andreas. (2015). Historical consciousness, historical competencies - and beyond? Some conceptual development within German history didactics. Deutsches Institut für Internationale Pädagogische Forschung, Hamburg. http://www.pedocs.de/volltexte/2015/10811/pdf/Koerber_2015_Development_German_History_Didactics.pdf.

Leiva, Fernando. (2008). Latin American Neostructuralism. The Contradictions of Post-Neoliberal Development. University of Minnesota Press.

Levesque, Sthepane. (2008). Thinking historically: Educating students for the twenty-first century. University of Toronto Press.

Ministerio de Educación Pública. (2015). Educar para una nueva ciudadanía: Fundamentación de la transformación curricular costarricense. http://www.idp. mep.go.cr/sites/all/files/idp_mep_go_cr/publicaciones/7-2016_educar_para_ una_nueva_ciudadaniafinal.pdf. 
Ministerio de Educación Pública. (2016). Programas de estudio de Estudios Sociales Tercer Ciclo de la Educación General Básica y Educación Diversificada. Gobierno de Costa Rica, San José. https://www.mep.go.cr/sites/default/files/ programadeestudio/programas/esociales3ciclo_diversificada.pdf.

. (2017). Política educativa. La persona: Centro del proceso educativo y sujeto transformador de la sociedad. https://www.mep.go.cr/sites/default/ files/page/adjuntos/politicaeducativa.pdf.

Pagés, Joan. (2009). Enseñar y aprender ciencias sociales en el siglo XXI: Reflexiones casi al final de una década. Investigación en Educación, Pedagogía y Formación Docente, II Congreso Internacional (Libro 2, pp. 140-154) Universidad Pedagógica Nacional, Universidad de Antioquia, Corporación Interuniversitaria de Servicios.

Pinar, William. F. (2015). Educational experience as lived: Knowledge, history, alterity: the selected writings of William F. Pinar. Routledge.

Plá, Sebastián. (2014). Calidad educativa y didáctica de la historia en los gobiernos neoconservadores en México 2000-2012. Revista Tempo e Argumento, 6(11), 162-192. http://dx.doi.org/doi:10.5965/2175180306112014162.

(2018). Calidad educativa : Historia de una política para la desigualdad. UNAM/IISUE.

Plá, Sebastián., \& Pagés, Joan. (2014). La investigacion en la enseñanza de la historia en America latina. Bonilla Artigas.

Programa Estado de la Nación en Desarrollo Humano Sostenible. (2019). Séptimo Informe Estado de la Educación. https://www.uned.ac.cr/viplan/images/cppi/ documentos/ESTADO-EDUCACION-2019-WEB.pdf

Programa Estado de la Nación-BID. (2018). Costa Rica: El estado de las políticas públicas docentes. https://observatorioeducacion.org/sites/default/files/el-estado-de-politicas-publicas-abril-15.pdf.

Ross, E. Wayne., Mathison, Sandra., \& Vinson, Kevin. D. (2014). Social Studies Curriculum and Teaching in the Era of Standardization. En E. W. Ross (Ed.), The Social Studies Curriculum. Purposes, Problems, and Possibilities (pp. 25-50). SunyPress.

Thornton, Sthepen. J. (2017). A Concise Historiography of the Social Studies. En M. McGlinn, \& C. Mason (Edits.), The Wiley Handbook of Social Studies Research (pp. 9-41). John Wiley \& Sons, Inc.

Young, Michael. (2010). The future of education in a knowledge society: The radical case for a subject-based curriculum. Journal of the Pacific Circle Consortium for Education, 22(1), 21-32. 